\title{
DE CÓMO SE REPRODUCEN LOS GAZNÁPIROS
}

Raúl Albanece

Instituto Superior de Perfeccionamiento y Especialización Docente, Argentina

\section{INTRODUCCIÓN}

“¿Es éste un país de gaznápiros?” Norberto Firpo me sorprendió con esta pregunta que daba título a su columna Rigurosamente incierto el sábado 12 de junio de 2004, desde la página 25 del diario La Nación, de Buenos Aires, para inmediatamente responder en el primer párrafo del artículo "no, lo que pasa es que abundan en demasía." Firpo enumera algunos ejemplos entre los que menciona "todo estudiante olímpicamente burro, creído de que la vida es un videogame" y luego asevera que "son ellos el producto de padres gaznápiros [...] La palurdez de los hijos se hereda de los padres, casi siempre."

Desde que leí ese artículo no he dejado de pensar en él. Ahora, yo me pregunto: Si hay tanta abundancia de gaznápiros ¿Qué hace la sociedad para revertir esta situación?

La Real Academia de la Lengua define gaznápiro como "Palurdo, simplón, torpe, que se queda embobado con cualquier cosa." Lo que me lleva a pensar en mi experiencia como profesor en un Instituto de Formación Docente en el cual observo que los estudiantes tienden a un pensamiento bancario recepción, acumulación y repetición de conocimientos- y tienen escaso interés en resolver tareas que demandan cierto esfuerzo mental.

En los pasados cuatro años he notado en los alumnos de carreras de formación docente una creciente dificultad para integrar conocimientos y resolver problemas en forma autónoma, no consiguen identificar las ideas principales de un texto y se quedan "embobados con cualquier cosa" que sirva como ejemplo sin poder extraer la teoría subyacente. ¿Es esta dificultad resultado de un currículo con asignaturas aisladas en extremo? ¿de un uso inadecuado de los métodos de enseñanza? ¿o los estudiantes de ahora no prestan atención y prefieren las cosas semirresueltas para evitar esfuerzos intelectuales?

El avance de la globalización impone nuevos desafíos; especialmente a la Educación Superior en lo atinente a la formación integral de "profesionales versátiles y altamente capacitados en un contexto globalizado del conocimiento, comercio, intercambios culturales, y migración”. (Kehn, 1995:142). Un aspecto fundamental en la formación integral de los estudiantes es el desarrollo de su pensamiento crítico, el cual posibilita la independencia cognoscitiva... y como consecuencia, la reducción de la cantidad de gaznápiros que pululan por el país.

En este punto cabe preguntarse: si los jóvenes son producto de padres gaznápiros... ¿qué ocurre si esos padres se desempeñan como docentes? Como dice José Giannotti "en la competencia para encontrar un lugar bajo el sol, es más importante fingir que saber efectivamente hacer, porque siempre está la esperanza de que el hacer se aprenda haciendo." (Giannotti, 1987:44-45). 
Pues bien, a lo largo de este trabajo pretendo reflexionar sobre este hecho y tratar de dilucidar cómo es que se reproducen los gaznápiros y por dónde cortar el círculo vicioso.

\section{EL PROBLEMA DEL CURRÍCULO}

El currículo es un conjunto de prácticas educativas que se desarrollan durante un tiempo determinado para que los miembros jóvenes de una sociedad adquieran ciertas competencias reconocidas socialmente como necesarias.

Intentemos desmenuzar esta definición para poder comprenderla en toda su plenitud:

Primero nos dice que es un conjunto de prácticas educativas, es decir, los métodos utilizados para "desarrollar o perfeccionar las facultades intelectuales y morales del niño o del joven por medio de preceptos, ejercicios, ejemplos, etcétera" (DRAE). Por lo tanto el currículo predeterminaría los métodos de enseñanza que el docente ha de usar. Este punto lo analizaremos con mayor detenimiento en el próximo capítulo.

Luego agrega que estas prácticas educativas se desarrollan durante un tiempo determinado, normalmente, al diseñar el currículo se toma cierta unidad de tiempo y se determina cuántas unidades son necesarias para que los jóvenes adquieran los conocimientos y se relacionan estos conocimientos con las unidades de tiempo dando como resultado los "contenidos" a enseñar.

En tercer lugar nos especifica que la finalidad es que los miembros jóvenes de una sociedad adquieran ciertas competencias reconocidas socialmente como necesarias, de aquí se colige que el currículo sería un instrumento de socialización. Lo sea o no, indudablemente, el currículo debe reflejar la opinión de la sociedad en su conjunto ya que éste, en cierto modo, depende de principios sociales que lo regulan.

En lo que respecta al tiempo tenemos dos aspectos o niveles para analizar. En un nivel general, hablar de tiempo es hablar de la duración de la carrera. Aquí entran en discusión temas como carreras cortas, carreras largas, división entre carrera de grado y de posgrado, o si se debe entregar alguna certificación o titulación intermedia, por ejemplo. En un nivel más particular, hablar de tiempo implica hablar del tiempo acordado para cada asignatura, e incluso, hilando más fino, el tiempo acordado para cada contenido.

En este nivel particular de análisis debemos tomar en cuenta lo que Phillip Jackson llama "currículo oculto" y que Michael Apple define como "las normas y valores que son implícitas, pero eficazmente, enseñadas en la escuela y de las que no suele hablarse en las declaraciones de fines $u$ objetivos de los profesores." (Apple, 1986:113). Y no olvidarse de lo que Eisner llama "currículo cero", aquello que no fue incluido en el currículo.

El currículo oculto y el currículo cero están determinados por factores sociales que analizaremos más adelante. 
Según Henry Wasser hay una escuela de pensamiento que sugiere el dominio de un nuevo concepto de educación superior: carreras cortas no universitarias. Ambas estructuras —universitaria y no universitaria- tienen orígenes diferentes. Los centros educativos de educación superior no universitaria pueden haberse desarrollado a partir de instituciones de enseñanza enfocada en la práctica o pueden haber sido creados con una explícita filosofía de combinar entrenamiento práctico y educación teórica. Muchas universidades han enfrentado este desafío integrando más programas educativos enfocados en la práctica en sus propios currículos. (Cfr. Wasser, 1996:5).

Más adelante agrega que "las naciones de Europa central y del este piensan que esas estructuras son significativas en tanto se adaptan a un amplio ingreso y a la economía de mercado. [...] Las ventajas que se les atribuyen son: menores costos de programa como reflejo de la más corta duración de los cursos, menores cifras de deserción, menores desembolsos anuales por estudiantes y gran diversidad de cursos y programas. Además, ofrecen oportunidades de entrenamiento que responden flexiblemente a las demandas del mercado laboral. Los riesgos vienen cuando estas instituciones son percibidas como de segunda con el consecuente descontento de los estudiantes." (Wasser, 1996:6).

En Argentina, normalmente, este tipo de instituciones se conciben como Institutos de Formación Docente. Sólo recientemente se las está pensando como Institutos de Tecnicaturas, pero siempre asociado a la idea de continuar estudios en Universidades luego de la graduación como técnico. De hecho un sin número de institutos no universitarios están realizando convenios con universidades "para darle más nivel a las carreras", aceptando implícitamente que los estudios de nivel superior no universitarios son de baja calidad.

A mediados de la década del noventa, participé en el equipo que reformó la Escuela Municipal de Artes Visuales de la ciudad de Gualeguaychú para transformarla en el Instituto Superior de Artes. La meta era transformar una serie de talleres de educación netamente práctica en un instituto de educación superior no universitario con carreras de no más de tres años que combinaran la práctica con la teoría necesaria para darle sustento. Grande fue nuestra sorpresa cuando luego de más de un año de trabajo, en el Consejo General de Educación de la provincia se negaban a darle reconocimiento al nuevo currículo porque no estaba contemplado en el mismo el otorgamiento de títulos docentes. En la concepción de la educación artística de los funcionarios que evaluaban los currículos sólo era posible la formación docente o una licenciatura. En su forma de pensar, el Instituto Superior de Artes debía formar docentes o nada, los artistas se formaban en una universidad.

Luego de cuatro años de arduas negociaciones, tras un cambio de gobierno con la consiguiente renovación de altos funcionarios, se aprobó el nuevo currículo en septiembre de 2000. Este currículo pretendía integrar estudiantes con diferentes prerrequisitos y habilidades en un mismo curso y adaptarse a un modelo vocacional.

Según Wasser un "modo de categorización del segundo sector de la educación superior fue:

1) Modelo binario (Gran Bretaña y Australia en la década del sesenta). [...] Idéntico al de la universidad en términos de prerrequisitos de admisión, duración del curso y tipo de grado. [...] Eran, de hecho, universidades menores. [...] 
2) Modelo de etapas (EE.UU., Japón, Francia). [...] Programas no universitarios servirían como capacitación en su propio derecho o como primera etapa de un grado universitario. Este modelo perdió su momento en Europa en 1988 cuando la Comunidad Europea arregló el cumplimiento de tres años de estudios como la capacitación básica como entrada a las profesiones.

3) Modelo vocacional. En algunos países, escuelas de formación vocacional fueron elevadas de nivel preservando sus caracteres específicos. Se enfatiza la alta calidad manteniendo la identidad claramente diferenciada de la universidad. [...]

4) Modelo de integración comprensiva. [...] Muchas estructuras de educación postsecundaria están en un sistema integrado. Tienen un mismo gobierno, director y vicedirector, reglas de admisión y asignación de presupuesto." (Wasser, 1996:9).

Según las relaciones entre bs contenidos, Basil Bernstein distingue dos tipos de currículo: el agregado y el integrado. El currículo agregado es aquel que presenta los contenidos en forma aislada entre sí, un contenido agregado tras otro, en cambio, el currículo integrado presenta los contenidos interrelacionados entre sí.

Desde este punto de vista, el currículo de un sin número de instituciones de educación superior es agregado, lo que, a mi ver, genera la dificultad de integrar los conocimientos que tienen los estudiantes de hoy en día. Pareciera que los diseñadores de tales currículos presuponen que la capacidad de integrar conocimientos es algo innato en el ser humano, lo que hace innecesario enseñarlo en ninguno de los niveles educativos.

El vínculo entre contenidos es un principio regulador del currículo que subyace en los conceptos de clasificación y de enmarcación. "Clasificación, se refiere al grado de mantenimiento de los límites entre contenidos. [...] Enmarcación se refiere al rango de las opciones disponibles al maestro y al alumno en el control de lo que se transmite y recibe en el contexto de la relación pedagógica." (Bernstein, 1985:48-49). La relación entre ambos conceptos determina el tipo de código de conocimiento educativo que, como decíamos con respecto al currículo, puede ser agregado o integrado.

"La naturaleza de la clasificación y de la enmarcación afecta la estructura de poder-autoridad que controla la diseminación del conocimiento educativo, y la forma del conocimiento transmitido. En esta forma los principios de poder y de control social se realizan a través de los códigos del conocimiento educativo y, a través de dichos códigos, entran en, y configuran la conciencia." (Bernstein, 1985:54). Esto se realiza fundamentalmente a través del currículo oculto, generalmente de manera subliminal aun para el docente, y del currículo cero... "de eso no se habla".

Los códigos agregados ordenan el conocimiento de tal manera que "sólo unos pocos experimentan en su interior la noción de que el conocimiento es permeable, que su ordenamiento es provisional, que la dialéctica del conocimiento es la del cierre y la apertura." (Bernstein, 1985:57).

Los códigos integrados se basan en una idea central como hilo conductor entre las asignaturas. "EI concepto relacionante enfocará su atención sobre la estructura profunda de cada materia antes que sobre 
su estructura superficial. Esto conducirá a efectuar un énfasis sobre, y una exploración de, los principios generales y los conceptos mediante los cuales tales principios son obtenidos." (Bernstein, 1985:61).

El currículo diseñado para el Instituto Superior de Artes de Gualeguaychú estaba basado en un código integrado. El concepto relacionante era la obra de arte, ésta era analizada desde los distintos puntos de vista posibles. Desde la Historia del arte se analizaba el período al que pertenecía y su contexto sociocultural; desde la Estética se analizaba la filosofía del arte vigente en su momento y su relación con la actual; en los talleres se estudiaba y practicaba las técnicas empleadas en las realizaciones de dicho período; en Diseño y composición se analizaban las reglas compositivas de cada época; y por último en el taller de producción el alumno debía realizar una obra de arte propia empleando los conocimientos adquiridos en las distintas asignaturas.

Este modelo funcionó casi a la perfección el primer año de implementación, pero el segundo año surgieron problemas de coordinación con el ingreso de nuevos profesores, algunos de ellos con escasa experiencia al frente del aula, que se negaban a poner en práctica nuevas teorías o teorías que no habían sido estudiadas o enseñadas en sus carreras como una alternativa posible... es decir, pretendían repetir el modelo educativo en el cual se habían formado, justamente todo lo contrario de lo que pretendía hacer el equipo de diseñadores del nuevo currículo.

Cambio de gobierno mediante, las nuevas autoridades argumentaron que el diseño curricular era inapropiado y conducía a la confusión de los alumnos, los que no podían, según la visión de los funcionarios, identificar correctamente las asignaturas. Por lo tanto, luego de una reestructuración curricular impuesta desde la Dirección de cultura se regresó a un código agregado de carácter disciplinar, donde se forman pintores, escultores, grabadores específicamente y ninguno puede transgredir las fronteras de la especialidad, negando las tendencias actuales en arte donde se es artista, sin fronteras para combinar las especialidades artísticas.

En esta era posmoderna, donde la inestabilidad es lo predominante, William Doll propone que el currículo debe ser posmoderno. "Los profesores y alumnos precisan ser libres, alentados, obligados a desarrollar su propio currículo en una interacción conjunta unos con otros. La orientación general de donde ellos parten -libros didácticos, guías de currículo, departamentos de educación estatal, organizaciones profesionales o tradiciones pasadas- precisa ser exactamente así: general, amplia, indeterminada." (Doll, 1997:179). La teoría debe emanar de la práctica, y no al revés.

Para Shirley Grundy "La teoría de los intereses constitutivos del conocimiento, propuesta por el filósofo alemán Jürgen Habermas, proporciona un marco para dar sentido a las prácticas curriculares. Se trata de una teoría sobre los intereses humanos fundamentales que influyen en la forma de constituir o construir el conocimiento." (Grundy, 1991:23).

Para Habermas, citado por Grundy, las formas más puras de placer se experimentan en la racionalidad, y "la forma de manifestarse la racionalidad determinará lo que un grupo social podrá distinguir como conocimiento." (Grundy, 1991:26).

Habermas distingue tres intereses cognitivos: 
1) El interés técnico, que orienta a las ciencias empírico-analíticas, pretende controlar el medio a través de acciones basadas en leyes con fundamento empírico.

2) El interés práctico, que orienta las ciencias histórico-hermenéuticas, pretende interpretar el medio para interactuar con él.

3) El interés emancipador, que orienta la crítica, pretende dar autonomía y responsabilidad al sujeto con base en la comprensión del conocimiento.

El currículo "es una construcción social. Es más, la forma y objetivos de esta construcción estarán determinados por intereses humanos fundamentales que suponen conceptos de personas y de su mundo." (Grundy, 1991:39). Según estos intereses podemos tener un currículo técnico, uno práctico o uno emancipador.

Un currículo técnico se orienta a un producto que, se supone, es el alumno. "El plan u objetivos de aprendizaje del curriculum se implementan, mediante la habilidad del profesor, para producir el aprendizaje buscado en el alumno." (Grundy, 1991:45). Es una visión reproductiva del currículo, como expresa Grundy. Es una idea iluminista y hasta conductista, diría yo.

Se plantean los objetivos con anterioridad, basados en un ideal a alcanzar; el profesor imparte los conocimientos que se supone el alumno debe tener para formar parte plenamente de la sociedad a la cual pertenece, y el producto final es una persona que ha acumulado saberes, la calidad del producto final depende de la cantidad de respuestas correctas que ese alumno sea capaz de dar. Es el pensamiento bancario, como lo llama Freire. "Los modelos fundamentales de la sociedad se mantienen por suposiciones ideológicas tácitas, por normas, si así lo prefiere, que no suelen ser conscientes, así como por el poder y el control económico. Estas normas sirven para organizar y legitimar la actividad de muchos individuos que, en su interacción, conforman un orden social." (Apple, 1986:116). Es el intento por perpetuar el poder hegemónico de la cultura dominante. Pido disculpas a los grandes pensadores que defienden este tipo de currículos por mi atrevimiento, pero a mí me suena a que "sólo somos un ladrillo más en la pared", me suena a perrito de Pavlov, me suena a programación de computadora, me suena a reproducción de gaznápiros.

El currículo técnico es el campo más fértil para el desarrollo del currículo oculto. "Postula una red de suposiciones que, una vez internalizadas por los estudiantes, establecen los límites de la legitimidad. [...] Estas suposiciones son obligatorias para los estudiantes, pues en ningún momento se elaboran o cuestionan. Por el hecho mismo de ser tácitas [...] aumenta su poder en cuanto que aspectos de la hegemonía." (Apple, 1986:117). Este modelo no permite la evolución de la sociedad, sino que la inmoviliza.

Un currículo práctico se orienta a la interpretación, es decir, a la construcción de significados. "Esto supone que la preocupación fundamental del profesor será el aprendizaje. [...] El aprendizaje supone [...] la construcción del significado. Así, el contenido del curriculum debería estimular la interpretación y el ejercicio del juicio a cargo tanto del alumno como del profesor." (Grundy, 1991:passim). Aquí ya no existe una respuesta correcta, sino una buena respuesta, pero ¿el alumno es capaz de aplicar esa buena respuesta en darle un significado al medio que lo rodea e interactuar con él? ¿cómo saberlo? ¿no será una versión 
posmoderna del pensamiento bancario? Con la internet, el alumno puede tener acceso a conocimientos que el profesor quizá ignora, y de esta forma reproducir una buena respuesta encontrada en un sitio web.

Un currículo emancipador "tenderá a la libertad en una serie de niveles. Ante todo, en el nivel de la consciencia, los sujetos que participan en la experiencia educativa llegarán a saber teóricamente y en términos de su propia existencia cuándo las proposiciones representan perspectivas deformadas del mundo -perspectivas que sirven a los intereses de dominación-y cuándo representan regularidades invariantes de la existencia. En el nivel de la práctica, el currículum emancipador implicará a los participantes en el encuentro educativo, tanto profesor como alumno, en una acción que trate de cambiar las estructuras en las que se produce el aprendizaje y que limitan la libertad de modos con frecuencia desconocidos." (Grundy, 1991:39). Seguramente que éste no es el modelo perfecto, pero es el mejor de los tres ya que, de una forma u otra, incluye a los otros dos. Es necesario observar el medio primero, luego es necesario interpretarlo, para llegar, por último, a su comprensión y obtener de esta manera la autonomía de pensamiento.

\section{EL PROBLEMA DE LOS MÉTODOS}

¿El currículo determina los métodos o los métodos determinan el currículo? La respuesta, obviamente, depende del punto de vista de quien vaya a contestar esta pregunta.

Para Bernstein, los principios subyacentes que configuran el currículo, también configuran los métodos de enseñanzaaprendizaje. Como veíamos anteriormente "la naturaleza de la clasificación y de la enmarcación afecta la estructura de poder-autoridad que controla la diseminación del conocimiento educativo, y la forma del conocimiento transmitido." (Bernstein, 1985:54).

Bernstein desarrolla la siguiente clasificación del código del conocimiento educativo (Bernstein, 1985:50):

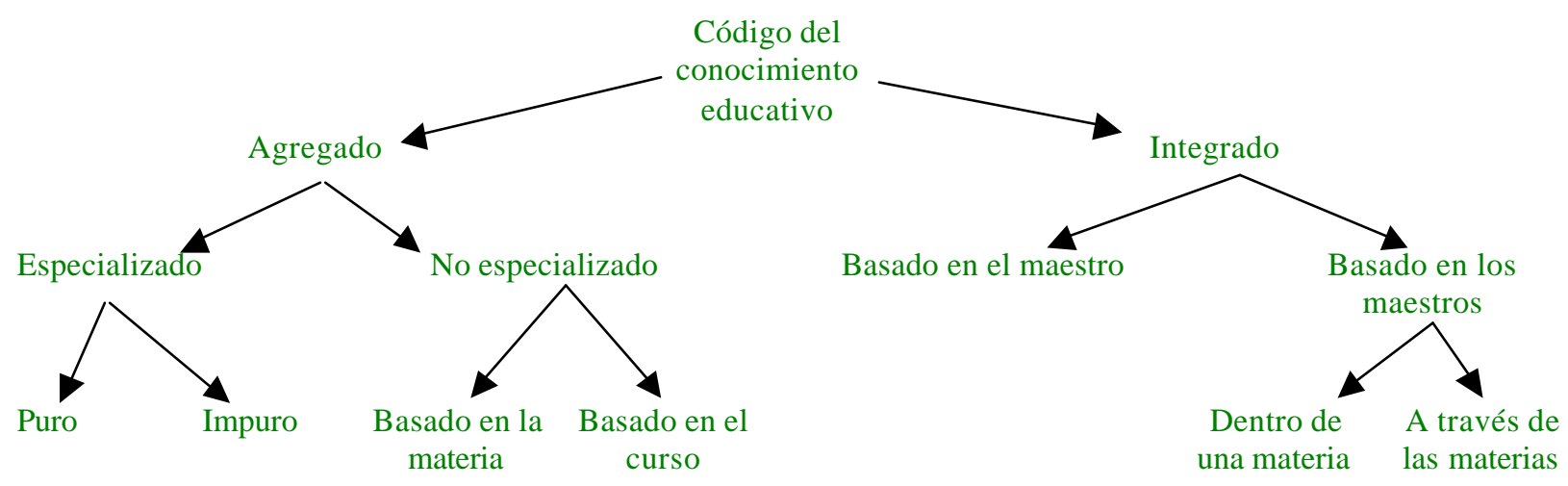


"La versión especializada del código agregado desarrolla procedimientos cuidadosos de selección para ver quien pertenece y quien no pertenece, y una vez tal selección ha tenido lugar, es muy difícil cambiar una identidad educativa. [...] La estructura profunda del tipo especializado del código agregado es el mantenimiento de la delimitación fuerte que produce el control desde dentro, a través de la formación de identidades específicas. [...] Con la aparición de nuevas formas de conocimiento surgen problemas críticos tales como el de su legitimidad, a qué instancia las formas pertenecen, cuándo, dónde y por qué tales formas deben ser enseñadas." (Bernstein, 1985:56).

Sin lugar a dudas es un código que no se condice con los tiempos posmodernos. El acelerado avance de las investigaciones en todos los ámbitos genera una inestabilidad permanente de las formas de conocimiento, por lo cual la versión especializada del código agregado permanentemente estaría enfrentando los problemas críticos descriptos por Bernstein. Los alumnos formados con este código egresan con conocimientos que, probablemente, ya estén superados ampliamente al momento que deban hacer uso de ellos; sin mencionar que, al menos en apariencia, los estudios interdisciplinarios no tienen cabida en este sistema.

En la versión no especializada del código agregado basada en la materia implica "aceptar una selección dada, una organización, un ritmo y una temporalización del conocimiento realizada en el marco pedagógico." (Bernstein, 1985:57). Este sistema hace hincapié en la adquisición del conocimiento y no en las formas de conocer; parte de un concepto iluminista desarrollado entre finales del siglo XVIII y principios del XIX. Este concepto asocia la cultura con la acumulación de saberes, por lo cual, quien sabe mucho sobre algún tema es popularmente considerado como culto.

El peligro de este sistema es suponer que existen sectores poblacionales más evolucionados o superiores que otros. El concepto iluminista afirma que todos los pueblos y todos los hombres deberían llegar, tarde o temprano, a adquirir las pautas y valores de la cultura dominante. Justamente ésta fue la idea que justificó la colonización europea y hoy da sustento a una globalización salvaje, antidemocrática y asimétrica.

"Los códigos integrados hacen mucho énfasis en las diversas formas de conocer en la relación pedagógica. [...] Los códigos integrados hacen accesible al alumno, desde el comienzo de su carrera educativa, [...] los principios para la generación de nuevo conocimiento." (Bernstein, 1985:61). Este sistema necesita de profesores altamente capacitados y fundamentalmente versátiles. Aplicar sistemas integrados genera resistencias, principalmente, de aquellos gaznápiros que ejercen la docencia de los que hablábamos en el prólogo.

Para Bernstein, "el orden creado por los códigos integrados puede ser problemático porque:

1) Debe haber consenso acerca de la idea integradora y ésta debe ser suficientemente explícita. [...] Los códigos integrados a nivel superficial crean límites débiles o no marcados, pero a nivel profundo descansan sobre ideologías cerradas explícitas. Cuando tales ideologías no son compartidas, las consecuencias se hacen visibles y amenazan la totalidad del código a cada momento.

2) La naturaleza de la unión entre la idea relacionante y el conocimiento que se coordina debe ser también coherentemente explicitada. [...] El código agregado es fácil de trabajar cuando el personal docente está constituido por maestros mediocres mientras que los códigos integrados 
demandan mucho mayor poder de síntesis, analogía y más habilidad tanto para tolerar como para gozar de la ambigüedad a nivel del conocimiento y de las relaciones sociales.

3) Debe constituirse un comité entre el personal docente para crear un mecanismo sensible de retro-información; el comité también actuará como agencia de socialización dentro del código. $[\ldots]$

4) Una de las mayores dificultades inherentes a los códigos integrados surge de aquello que debe ser evaluado así como de la forma de evaluación, y del lugar que ocupan las competencias específicas en tal evaluación." (Bernstein, 1985:65 y ss.).

La temporalización de los contenidos es un factor del currículo oculto pocas veces tenido en cuenta. La valoración de los contenidos, subliminalmente, se transmite a los alumnos en el tiempo que se dedica a ciertos temas. A mayor valoración, mayor tiempo dedicado a ese contenido; y aquí entra el currículo cero de Eisner, es decir, aquello a lo que no se le dedica tiempo alguno, aquello que ni siquiera se menciona se encuentra en el último lugar en la escala de valores de esa sociedad.

Dreeben, citado por Apple, "afirma que los estudiantes aprenden tácitamente determinadas normas sociales identificables sobre todo al enfrentarse a las tareas y dificultades cotidianas de la vida en el aula Es decisivo el hecho de que las normas que aprenden los estudiantes penetren en muchas áreas de la vida posterior, pues ayudan a documentar de qué modo contribuye la enseñanza al ajuste individual a un orden político, económico y social continuado." (Apple, 1986:117-118).

Más adelante, Apple agrega que durante la escolarización se hace énfasis "en los estudiantes -y en el hombre en general- en cuanto que personas transmisoras y receptoras de valores, y no como personas creadoras de valores. [...] La base subyacente del paradigma social utilizado para guiar y ordenar nuestras percepciones se orienta fundamentalmente a la legitimación del orden social existente."(Apple, 1986:125).

El diseño curricular basado en el interés técnico de Habermas exige métodos lineales orientados al producto. "Los objetivos son enunciados o principios teóricos que se encuentran en una relación determinista con el mundo de la práctica. [...] El producto de un proceso de aprendizaje diseñado de este modo se juzgará de acuerdo con la fidelidad con que la puesta en práctica del diseño cumpla los objetivos, produciendo, por tanto, el resultado deseado." (Grundy, 1991:50).

Un currículo técnico presupone un modelo vertical de poder, cuyo poder mayor se centra en quien formula los objetivos. La formación del docente con un currículo de estas características "se considera un entrenamiento del profesor, de modo que el curriculum de formación docente comprende el aprendizaje de un conjunto de métodos mediante los que se llevará acabo la acción docente." (Grundy, 1991:56). Esto sería como memorizar una serie de recetas infalibles que se utilizarán en la labor profesional, sin posibilidad alguna de variar de métodos. Todo contenido a enseñar debe transformarse en un producto medible objetivamente, de lo contrario carece de validez en este contexto netamente positivista.

Un currículo práctico presupone un modelo menos vertical que el técnico, pero no llega a ser plenamente horizontal, es un modelo oblícuo, podríamos decir. Éste tipo de currículos permite libertad al docente en cuanto a su interpretación. "Esta perspectiva supone que el práctico no sólo tiene derecho, sino también obligación, de dar su propio significado al texto." (Grundy, 1991:100). Asumir esta postura, compromete al docente a ver al alumno como sujeto activo del proceso de enseñanza-aprendizaje, con el 
mismo derecho y obligación de dar su propio significado al texto. Aprender, en este contexto, implica interpretar el conocimiento. "El acto de atribución de significado constituye un acto de interpretación y la práctica de la interpretación de textos literarios puede sernos útil al respecto." (Grundy, 1991:98).

Stenhouse, citado por Grundy, afirma que "el ideal es que la especificación del conocimiento aliente una investigación y un programa de desarrollo personales por parte del profesor, mediante el cual éste aumente progresivamente la comprensión de su propia labor y perfeccione así su enseñanza." (Grundy, 1991:103).

Desde este punto de vista el currículo es un proceso permanente de construcción. El currículo es el punto de partida y no el punto de llegada como lo es el currículo técnico.

Un currículo emancipador, como decía en el capítulo anterior, es el mejor de los tres modelos. Para que el alumno alcance su autonomía, los métodos de enseñanza-aprendizaje deben basarse en la forma de construir el conocimiento. No debe darse al alumno el conocimiento sino las maneras de encontrarlo por sí mismo. Esta perspectiva implica que deben formarse investigadores; ve a los alumnos como personas creadoras de valores y constructoras del conocimiento.

El interés práctico y el emancipador, ven al currículo como proceso. Por lo tanto los contenidos pueden variar de acuerdo con los intereses de cada sujeto. El peligro radica en la posibilidad de caer en un currículo basado en las necesidades del alumno. "Según la forma en que interpretemos necesidad, el programa de acción propuesto resultará en algunos casos trivial, en otros indeterminado o sin fundamento, pero siempre poco importante." (Othanel Smith - Ennis, 1971:42).

\section{EL PROBLEMA DE LOS ALUMNOS}

Como decía en la introducción, observo que los estudiantes tienden a un pensamiento bancario y tienen dificultad para integrar conocimientos y resolver problemas en forma autónoma. Pero, como siempre repito, no son culpables, sino víctimas del sistema.

Si los jóvenes de hoy son gaznápiros, lo son porque los hicieron así; ya sean sus padres como postula Firpo o sean sus primeros maestros.

Si han sido sus primeros maestros, éstos pueden haber sido víctimas del sistema también, puesto que si el diseño curricular es técnico los maestros no tienen demasiada ingerencia en el desarrollo del mismo y sólo deben limitarse a reproducir recetas infalibles para que el alumno asimile los conocimientos. Tanto el currículo técnico como el agregado generan la dificultad de integrar los conocimientos que tienen los estudiantes de hoy en día. Como dije anteriormente, pareciera que los diseñadores de tales currículos presuponen que la capacidad de integrar conocimientos es algo innato en el ser humano, lo que hace innecesario enseñarlo en ninguno de los niveles educativos.

El acelerado avance de los conocimientos hace que la escolaridad se extienda en el tiempo, y que sean necesarios -y hasta obligatorio- los estudios de nivel superior aún para el puesto de trabajo más sencillo. Esto hace que se incremente la población estudiantil en las instituciones educativas de nivel superior universitarias y no universitarias. 
Cualquier sociedad democrática y, fundamentalmente aquellos países en vías de desarrollo deben asegurar a los jóvenes el acceso irrestricto a la educación superior. Pero si el sistema educativo no funciona en óptimas condiciones, se hace necesario tomar exámenes de ingreso.

Una certificación de estudios completos, cualquiera sea el nivel, debería acreditar ciertas competencias. Sin embargo, en nuestro país no sucede así. Numerosos alumnos ingresan año a año en instituciones de educación superior sin siquiera poder extraer las ideas principales de un texto. Probablemente si se institucionalizaran los exámenes de ingreso, ninguno de ellos tendría acceso a la educación superior, sólo basta leer los diarios que dan cuenta de los masivos aplazos en los exámenes de ingreso en distintas facultades. Antes de culpar a los jóvenes de no estudiar preguntemos cómo obtuvo su certificado de EGB si justamente esa Educación General Básica no la posee o no le fue impartida como corresponde.

Como dice José Giannotti, "Delante de esa multitud de alumnos se colocan profesores mal pagos, recibiendo en general por hora-cátedra, dedicándose marginalmente a la docencia [...]; profesores titularizados más por trucos de legislación que por haber recorrido las agruras de los concursos; profesores que ni siquiera publicaron un artículo, que pasaron a las escondidas sus tesis; profesores que obtuvieron sus diplomas no se sabe cómo y que ahora [...] están ocupando importantes posiciones de mando; en fin, toda esa masa de docentes formando un bajo clero connivente con la viveza y que, nunca, de modo alguno, estará dispuesta a jugar las reglas de la competencia y de la evaluación recíproca." (Giannotti, 1987:52). Si en la sociedad argentina abundan los gaznápiros, es lógico que abunden en los distintos grupos que la conforman. Si tenemos claustros con abundancia de gaznápiros, es natural que se reproduzcan en proporción geométrica.

\section{CONCLUSIÓN}

¿Cómo enfrentar este problema? ¿Cómo evitar que los gaznápiros sigan abundando en el país? Principalmente aislando al vivo o avivado que prefiere parecer antes que ser.

"Teniendo siempre presentes experiencias vividas en otras partes del mundo, procuremos resolver los problemas desde nuestro punto de vista. $Y$ de lo que más carecemos no es propiamente de conocimientos, sino de la práctica cotidiana de ellos." (Giannotti, 1987:47-48).

Según Barbara Kehm un egresado para actuar en el futuro debe ser capaz de:

1) Generar, identificar y definir problemas y plantear cuestionamientos serios.

2) Pensar y encontrar soluciones razonables a los problemas.

3) Utilizar tecnología.

4) Actuar con autonomía individual en situaciones complejas.

5) Participar efectivamente en la sociedad del nuevo siglo y realizar elecciones éticas.

6) Devolver la coherencia, y de conceptualizar tanto como integrar abordajes conceptuales y conocimientos dispares de manera de transformarlos en útiles, aplicables y comprensibles. 
7) Esforzarse en trabajar por un mundo nuevo y mejor, guiado por la interrelación entre conocimiento objetivo, propósitos morales y práctica social.

8) Construir una relación inteligente entre el público y él. (Cfr. Kehm, 1995:145).

Para ello es necesario que se eduque en base a un currículo integrado, que el alumno tenga la oportunidad de darse cuenta de que la naturaleza es una sola y cada objeto de estudio tiene la posibilidad de ser estudiado desde múltiples puntos de vista. Para que el alumno adquiera autonomía es necesario que el currículo, siguiendo la postura de Habermas citado por Shirley Grundy, se inspire en un interés emancipador. Como dije con anterioridad, éste no es el modelo perfecto, pero es el mejor de los que tenemos hasta el momento.

"Combatir el provincianismo, sin embargo, significa tramar una tradición autorreferente, lo que implica formar un público que no sea exclusivamente de especialistas. De ahí la necesidad de trabajar en dos niveles; de un lado, el plano académico propiamente dicho, con sus rituales y tics; de otro, el debate cultural e ideológico, cuando la posición del investigador toma el rostro de un formador de opinión." (Giannotti, 1987:54).

Claro que esto no se logra con sólo desearlo, es necesario algunos años de transformación del sistema educativo en su totalidad, pero contrariamente a como se han hecho las transformaciones hasta hoy, es necesario empezar por arriba, es decir, empezar en el nivel superior, fundamentalmente en la formación docente pues esos nuevos docentes generarán un cambio en sus alumnos y de esta manera se transformará la sociedad en su conjunto "porque reformar la universidad significa reformar también la sociedad." (Kehm, 1995:141). Y quizá, poco a poco, dejen de reproducirse los gaznápiros.

\section{BIBLIOGRAFÍA}

APPLE, Michael. Ideología y currículo. Madrid, Akal Universitaria. 1986.

BERNSTEIN, Basil. "Clasificación y enmarcamiento del conocimiento educativo" en Revista Colombiana de Educación. (№ 15) 1985. Págs. 45-71.

DoLL, William (jr.). Currículo: Uma perspectiva pos-moderna. Porto Alegre, Artes Médicas. 1997.

FIRPO, Norberto. “¿Es éste un país de gaznápiros?” en diario La Nación de Buenos Aires, sábado 12 de junio de 2004. Pág. 25.

GIANOTTI, José Arthur. A Universidade em Ritmo de Barbarie. São Paulo, Brasiliense. 1987.

GRUNDY, Shirley. Producto o praxis del curriculum. Madrid, Morata. 1991.

KEHN, Barbara. (1995) "Implementing Reforms or Conceptualizing the University of the Future?" en Tertiary Education and Management. (Vol. 1, № 2) 1995. Págs. 140-147.

OTHANEL SMITH, B. - Robert H. ENNIS. Lenguaje y conceptos en educación. Buenos Aires, El Ateneo. 1971.

WASSER, Henry. (1996) "Short-Cycle Higher Education" en Tertiary Education and Management. (Vol. 2, № 1) 1996. Págs. 5-9. 


\title{
Contactar
}

Revista lberoamericana de Educación

\author{
Principal OEI
}

